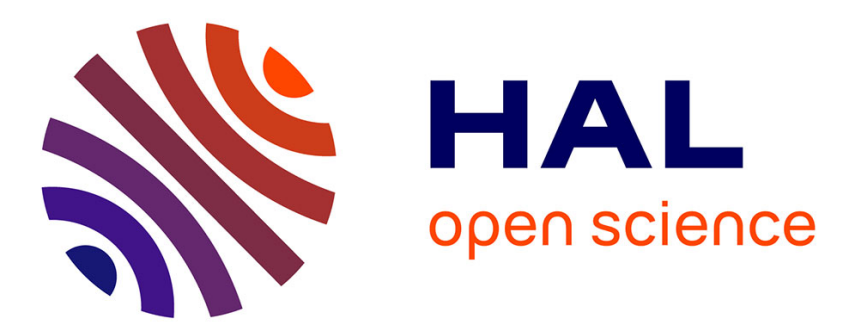

\title{
Low-complexity detector for very large and massive MIMO transmission
}

\author{
Yasser Fadlallah, Abdeldjalil Aissa El Bey, Karine Amis Cavalec, Dominique
}

Pastor

\section{- To cite this version:}

Yasser Fadlallah, Abdeldjalil Aissa El Bey, Karine Amis Cavalec, Dominique Pastor. Low-complexity detector for very large and massive MIMO transmission. SPAWC 2015: 16th IEEE International Workshop on Signal Processing Advances in Wireless Communications, Jun 2015, Stockholm, Sweden. pp.251 - 255, 10.1109/SPAWC.2015.7227038 . hal-01203097

\section{HAL Id: hal-01203097 \\ https://hal.science/hal-01203097}

Submitted on 22 Sep 2015

HAL is a multi-disciplinary open access archive for the deposit and dissemination of scientific research documents, whether they are published or not. The documents may come from teaching and research institutions in France or abroad, or from public or private research centers.
L'archive ouverte pluridisciplinaire HAL, est destinée au dépôt et à la diffusion de documents scientifiques de niveau recherche, publiés ou non, émanant des établissements d'enseignement et de recherche français ou étrangers, des laboratoires publics ou privés. 


\title{
Low-complexity Detector for Very Large and Massive MIMO Transmission
}

\author{
Yasser Fadlallah Student Member, IEEE Abdeldjalil Aïssa-El-Bey Senior Member, IEEE, Karine \\ Amis Member, IEEE, and Dominique Pastor Member, IEEE \\ Institut Télécom; Télécom Bretagne; UMR CNRS 6285 Lab-STICC \\ Technopôle Brest Iroise CS 8381829238 Brest, France \\ Université européenne de Bretagne \\ Email: Firstname.Lastname@ $@$ telecom-bretagne.eu
}

\begin{abstract}
Maximum-Likelihood (ML) joint detection has been proposed as an optimal strategy that detects simultaneously the transmitted signals. In very large multiple-input-multiple output (MIMO) systems, the ML detector becomes intractable due the computational cost that increases exponentially with the antenna dimensions. In this paper, we propose a relaxed ML detector based on an iterative decoding strategy that reduces the computational cost. We exploit the fact that the transmit constellation is discrete, and remodel the channel as a MIMO channel with sparse input belonging to the binary set $\{0,1\}$. The sparsity property allows us to relax the ML problem as a quadratic minimization under linear and $\ell_{1}$-norm constraint. We then prove the equivalence of the relaxed problem to a convex optimization problem solvable in polynomial time. Simulation results illustrate the efficiency of the low-complexity proposed detector compared to other existing ones in very large and massive MIMO context.
\end{abstract}

\section{INTRODUCTION}

In wireless communications, multiple-input-multiple output (MIMO) transmission is a technology that significantly increases the achievable data rate without any extra transmission power [1]. Multi-user massive MIMO network is a scenario that has been recently proposed, where many mobile terminals are served by a Base Station (BS) equipped with a very high antenna number [2]. In such a scenario, the detection in the uplink remains a challenge, since the BS is required to detect signals transmitted from all users while trying to exploit full received diversity. The optimal detection criterion that fulfills the diversity requirement is the Maximum-Likelihood (ML) joint detection which has been proposed to detect jointly the transmitted signals [3]. ML detector is able to minimize the probability of error in the medium and high signal-to-noise ratio (SNR) region. However, such a criterion is not applicable to the addressed multi-user massive MIMO scenario due to its computational complexity that increases exponentially with the number of signals to be detected. Alternative solutions have been proposed aiming at reducing the complexity while preserving optimal performance. Among these solutions the sphere decoder (SD) based on a welldefined spherical search [4]. However, SD exhibits also a variable computational complexity that highly depends on the SNR region, the signal dimensions, and the sphere radius initialization. The computational complexity order has been upper-bounded by $\mathcal{O}\left(M^{\gamma N}\right)$, where $\gamma \in(0,1]$ depends on both SNR value and sphere radius initialization, $N$ is the signal dimension, and $M$ is the constellation size [5]. Other proposed linear solutions such as minimum mean square error (MMSE) and zero-forcing (ZF) present rather low complexity at the expense of a high performance-loss.

In order to suit high dimensions problems, this paper aims to find out a relaxed sub-optimal detection criterion that significantly reduces the complexity order. The key idea is to exploit the finite transmit constellation size, and to relax the ML problem constraints such that iterative algorithms can be applied. The iterative strategy of decoding aims to maintain a low computational cost even when the signal and/or constellation size increase. Firstly, the MIMO channel model with inputs belonging to a discrete transmit constellation is shown equivalent to a new model with sparse inputs belonging to the binary set $\{0,1\}$. Then, the ML detection problem is formulated as a minimization problem of a quadratic cost function under linear and constant $\ell_{0}$-norm constraints. However, such a problem is Non Polynomial (NP-hard) because of the constant $\ell_{0}$-norm constraint. Referring to the literature of sparse problems, and in order to reduce the computational cost, the $\ell_{0}$-norm can be relaxed by the $\ell_{1}$-norm even though the equivalence is not always true. This relaxation pushes the problem to have a solution at the intersection of a lozenge of unit diameter and an explicit plan. On the the other hand, the $\ell_{1}$-norm equality constraint can be readily proven as nonconvex constraint. In order to overcome this non-convexity, we prove that, thanks to the presented linear constraints in the new detection problem, the constant $\ell_{1}$-norm amounts to ensuring that all components of the variable vector are positive. As a result, the relaxed detection problem is a minimization of a quadratic function under linear equality and positive variables constraint. Such problems can be resolved using first order iterative algorithms (i.e. gradient descent) or even more accurate algorithms such as primal-dual interior point (PDIP) methods [6]. A main advantage over other low complexity detectors such as the MMSE Successive Interference Cancellation (MMSE-SIC) is that, the proposed detector does not require any modification when applied to underdetermined ${ }^{1}$ MIMO systems, which is not the case for the MMSE-SIC [7].

${ }^{1}$ When the number of receive antennas is smaller than the number of transmitted signals 


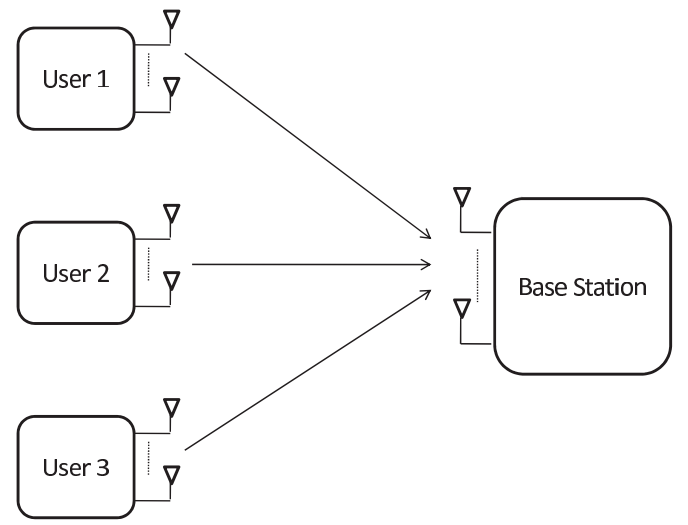

Fig. 1: 3-user MIMO multiple access channel

The rest of the paper is organized as follows. The system model is described in Section II. The new MIMO sparse model is introduced in Section III. Section IV proposes a relaxed ML problem that minimizes a quadratic objective function under linear equality and positive variables constraints. In Section V, the simulation results enable the evaluation of our contribution. Finally, Section VI concludes the paper.

Notations: The superscripts $(.)^{T}$ and $(.)^{H}$ stand for the transpose and transpose conjugate matrices, respectively. The norm $\ell_{p}$ is represented by $\|.\|_{p}$, and the Kronecker product by $\otimes . \boldsymbol{I}_{N}$ is the $N \times N$ identity matrix and $\mathbf{1}_{N}$ is the $N$-length vector with components equal to one.

\section{SYSTEM MODEL}

We consider a scenario where $K$ User Equipment (UE) equipped with $M_{k}$ antennas each, are communicating with a BS equipped with $N$ antennas, and $N \gg M_{j} \forall j \in$ $\{1, \cdots, K\}$. We assume a perfect channel state information (CSI) knowledge between the BS and all UEs at the BS. The received signal is defined as follows

$$
\boldsymbol{y}=\sum_{k=1}^{K} \boldsymbol{H}_{k} \boldsymbol{x}_{k}+\boldsymbol{z}=\overline{\boldsymbol{H}} \boldsymbol{x}+\boldsymbol{z},
$$

where $\overline{\boldsymbol{H}}=\left[\boldsymbol{H}_{1}, \cdots, \boldsymbol{H}_{K}\right] \boldsymbol{H}_{k}, \boldsymbol{x}=\left[\boldsymbol{x}_{1}^{T}, \cdots, \boldsymbol{x}_{K}^{T}\right]^{T}, \boldsymbol{H}_{k} \in$ $\mathbb{C}^{N \times M_{k}}$ is the random channel matrix between the $k^{\text {th }} \mathrm{UE}$ and the BS, $x_{k}$ is the $M_{k} \times 1$ data vector from the $k^{\text {th }} \mathrm{UE}$ with symbols selected from a finite alphabet constellation, and $\boldsymbol{z}$ is the $N \times 1$ circularly symmetric additive Gaussian noise vector with zero mean and covariance matrix equals to $\sigma^{2} \boldsymbol{I}_{N}$. The components of $\boldsymbol{x}_{k}$ belong to a finite alphabet constellation defined as $\mathcal{A}=\left\{a_{1}, \cdots, a_{L}\right\}$ where $L$ is the cardinal of the set $\mathcal{A}$. For example, the Binary Phase Shift Keying (BPSK) constellation is the set $\mathcal{A}=\{-1,+1\}$ of cardinal $L=2$.

\section{SPARSE DECOMPOSITION}

The goal of this paper is to propose an efficient detector, characterized by a polynomial complexity order with respect to the antenna dimensions, that jointly detects all transmitted signals at once. We assume a priori knowledge of the transmit constellation. We exploit the fact that the original symbols belong to a finite and discrete constellation, and we decompose each symbol on the basis of the vector space in which the finite alphabet vector can be cast i.e. $\boldsymbol{a}=\left[a_{1}, \cdots, a_{L}\right]$ where $L$ is the finite alphabet cardinal. That is, the joint symbol vector stacking all UEs transmitted data with $M$ entries $(M=$ $\sum_{j=0}^{K} M_{j}$ ), can be modeled as an equivalent sparse data vector with $M \times L$ entries. The $j^{\text {th }}$ symbol $x_{j}$ of $\boldsymbol{x}$ can be formulated as

$$
\begin{aligned}
x_{j} & =\boldsymbol{a} \boldsymbol{s}_{j}^{T}, \\
\text { where } \boldsymbol{s}_{j} & \left.=\left[\delta_{a_{1}}\left(x_{j}\right), \delta_{a_{2}}\left(x_{j}\right)\right), \cdots, \delta_{a_{L}}\left(x_{j}\right)\right],
\end{aligned}
$$

where $\delta_{a_{i}}\left(x_{j}\right)$ is the discrete delta measure which is equal to 1 when $a_{i}=x_{j}$ and 0 otherwise. Applying this decomposition over all symbols, the vector $\boldsymbol{x}$ can be expressed in function of a sparse vector $s$ as

$$
\begin{aligned}
\boldsymbol{x} & =\boldsymbol{B}_{\boldsymbol{a}} \boldsymbol{s}, \\
\text { where } \boldsymbol{s} & =\left[\boldsymbol{s}_{1}, \cdots, \boldsymbol{s}_{M}\right]^{T}, \text { and } \boldsymbol{B}_{\boldsymbol{a}}=\boldsymbol{I}_{M} \otimes \boldsymbol{a} .
\end{aligned}
$$

$\boldsymbol{B}_{\boldsymbol{a}}$ is a block diagonal matrix of size $M \times M L$. Substituting (3) into (1) yields the received signal as follows

$$
\boldsymbol{y}=\overline{\boldsymbol{H}} \boldsymbol{B}_{a} s+z .
$$

The sparsity degree ${ }^{2}$ of $s$ can be calculated as $L(M-1)$.

In order to better clarify the above decomposition, we give the following example. Let us consider a BPSK constellation with a basis vector $\boldsymbol{a}=[-1,1]$. Let us define $\boldsymbol{x}$ a vector with elements belonging to the BPSK constellation, and $x=[1,-1]^{T}$. Applying the decomposition in (2) and (3), each element of $\boldsymbol{x}$ can be cast on the constellation basis $\boldsymbol{a}$ such that $\boldsymbol{x}$ can be rewritten as

$$
\left(\begin{array}{c}
1 \\
-1
\end{array}\right)=\left(\begin{array}{cccc}
-1 & 1 & 0 & 0 \\
0 & 0 & -1 & 1
\end{array}\right) \cdot\left(\begin{array}{l}
0 \\
1 \\
1 \\
0
\end{array}\right)
$$

The sparsity degree of $s=\left[s_{1} ; s_{2}\right]$ is 2 .

The transmitted symbols in the new model are the binary components of the vector $s$. In the upcoming section, we propose a convex approximation of the ML detector based on the euclidean distance minimization to detect the new variable vector $s$ iteratively using low-complexity algorithms [6].

\section{RELAXED ML DETECTOR}

\section{A. Relaxed Minimization Problem}

The ML criterion seeks the solution with the closest point to the received signal in the received constellation, that is, the symbol vector that satisfies a minimum euclidean distance between $\boldsymbol{y}$ and $\overline{\boldsymbol{H}} \boldsymbol{x}$. The detection problem formulation is given by

$$
\left(P_{0}\right): \underset{\boldsymbol{x} \in \mathcal{A}^{M}}{\arg \min }\|\boldsymbol{y}-\overline{\boldsymbol{H}} \boldsymbol{x}\|_{2} .
$$

Such a problem suffers from a high computational cost due to the indispensable exhaustive search over the set $\mathcal{A}^{M}$. Using the symbol vector decomposition as in the previous section, we propose the following

\footnotetext{
${ }^{2}$ The sparsity degree of $s$ denotes the number of non-zero elements in $s$.
} 
Lemma 1: The minimization problem $\left(P_{0}\right)$ is equivalent to the following problem

$$
\begin{array}{cc}
\left(P_{1}\right): \quad \underset{s \in \mathrm{R}^{M L}}{\arg \min } & \left\|\boldsymbol{y}-\overline{\boldsymbol{H}} \boldsymbol{B}_{a} \boldsymbol{s}\right\|_{2} \\
\text { subject to } & \boldsymbol{B}_{1} \boldsymbol{s}=\mathbf{1}_{M},\|\boldsymbol{s}\|_{0}=M .
\end{array}
$$

where the $\ell_{0}$-norm is the weight of $s$ and the block diagonal $M \times M L$ matrix $\boldsymbol{B}_{1}$ is given by

$$
\boldsymbol{B}_{\mathbf{1}}=\boldsymbol{I}_{M} \otimes \mathbf{1}_{L}^{T} .
$$

Proof: Let us denote $\boldsymbol{x}$ a vector with $M$ elements belonging to a finite alphabet set. Referring to Section II, $\boldsymbol{x}$ can be decomposed as $\boldsymbol{x}=\boldsymbol{B}_{a} s$, where $s$ consists of $M$ sub-vectors with only one non-zero element equal to one. In other words, the sum over each sub-vector is equal to one i.e. $\boldsymbol{B}_{1} s=\mathbf{1}_{M}$, and the total number of non-zero elements in $s$ is equal to $M$ i.e. $\|s\|_{0}=M$. Let us now assume the following hypotheses: $\boldsymbol{B}_{1} s=\mathbf{1}_{M}$ and $\|s\|_{0}=M$. The first condition $\boldsymbol{B}_{1} s=\mathbf{1}_{M}$, i.e. $\sum_{p=1}^{L} s_{(j-1) L+p}=1$ for all $j \in\{1, \cdots, M\}$, implies that at least one non-zero element exists in any sub-vector $j \in\{1, \cdots, M\}$, with a minimum total non-zero elements number $M$. The second condition $\|s\|_{0}=M$ imposes the total non-zero elements number to be equal to $M$, thus along with the first condition, each sub-vector can contain only one element different from zero and equal to one. Thereby, the projection of the vector $s$ onto the dictionary matrix $\boldsymbol{B}_{a}$ yields a vector $\boldsymbol{x}=\boldsymbol{B}_{a} \boldsymbol{s}$ in the finite alphabet constellation $\mathcal{A}^{\mathcal{M}}$.

Solving a quadratic minimization problem under $\ell_{0}$-norm constraint is in general complex and may require exhaustive search strategy, which can be intractable in practice for large signal dimensions. Therefore, by mimicking literature on sparse reconstruction [8], we propose to replace the $\ell_{0}$-norm by the $\ell_{1}$-norm. The reason for this approximation is that the $\ell_{1}$-norm represents the best convex approximation of the the $\ell_{0}$-norm [9]. Thus, we address the much simpler problem $P_{2}$,

$$
\begin{aligned}
& \left(P_{2}\right): \quad \underset{\boldsymbol{s} \in \mathrm{R}^{M L}}{\arg \min } \quad\left\|\boldsymbol{y}-\overline{\boldsymbol{H}} \boldsymbol{B}_{\boldsymbol{a}} \boldsymbol{s}\right\|_{2}
\end{aligned}
$$

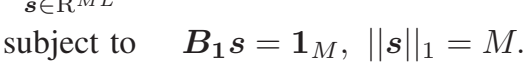

On the other hand, let $\mathcal{S}$ be the set defined by the constraint $\|s\|_{1}=M$, by referring to the definition of a convex set,

$$
\forall s_{1}, s_{2} \in \mathcal{S}, \lambda s_{1}+(1-\lambda) s_{2} \in \mathcal{S},
$$

we find out that the constraint $\|s\|_{1}=M$ does not defined a convex set. But it defines a convex set when it is combined with the constraint $B_{1} s=\mathbf{1}_{M}$. In order to prove our claim, we propose the following lemma

Lemma 2: Let us define a real vector $s$ of length $M L$ as given in Section III, and satisfying $\boldsymbol{B}_{1} s=\mathbf{1}_{M}$. The $\ell_{1}$-norm is equal to $M$ if and only if all elements of $s$ are positive.

Proof: Let $\boldsymbol{B}_{1}=\boldsymbol{I}_{M} \otimes \mathbf{1}_{L}^{T}$. The non-zeros elements of the $k^{\text {th }}$ row of $\boldsymbol{B}_{1}$ are all equal to one and are those whose indices range from $(k-1) L+1$ to $k L$. Thus $\boldsymbol{B}_{1} s=\mathbf{1}_{M}$ implies

$$
\sum_{p=1}^{L} s_{(k-1) L+p}=1, \quad \forall k \in\{1, \cdots, M\}
$$

By successive additions with respect to $k$, we obtain

$$
\sum_{i=1}^{M L} s_{i}=\sum_{k=1}^{M} \sum_{p=1}^{L} s_{(k-1) L+p}=M .
$$

Let us first assume that all components of $s$ are positive. Then $\boldsymbol{s}_{i}=\left|\boldsymbol{s}_{i}\right|$ and using (12), we deduce that $\sum_{i=1}^{M L}\left|s_{i}\right|=M$, i.e. $\|s\|_{1}=M$.

Let us now assume that $\|s\|_{1}=M$. According to (12), we can thus write

$$
\sum_{i=1}^{M L}\left(\left|s_{i}\right|-s_{i}\right)=0 .
$$

Let $\mathcal{N}(s) \neq \emptyset$ denote the set of indices corresponding to all nonzero negative elements of $s$. Then $\sum_{i=1}^{M L}\left(\left|s_{i}\right|-s_{i}\right)=$ $2 \sum_{i \in \mathcal{N}(s)}\left|s_{i}\right|$. It follows from (13) that $s_{i}=0$ for every $i \in \mathcal{N}(s)$ which is in contradiction with $\mathcal{N}(s) \neq \emptyset$. We thus deduce that $\mathcal{N}(s)=\emptyset$ and all components of $s$ are positive.

The set defined by the constraints $\boldsymbol{B}_{1} s=\mathbf{1}_{M}$ and $s \geq 0$ is obviously convex. Thence, the decoding problem becomes

$$
\begin{aligned}
& \text { [Quad-min] }: \underset{\boldsymbol{s} \in \mathrm{R}^{M L}}{\arg \min }\left\|\boldsymbol{y}-\boldsymbol{H} \boldsymbol{B}_{\boldsymbol{a}} \boldsymbol{s}\right\|_{2} \\
& \text { subject to } \boldsymbol{B}_{\mathbf{1}} \boldsymbol{s}=\mathbf{1}_{M}, \text { and } \boldsymbol{s} \geq 0 .
\end{aligned}
$$

This new optimization model is a quadratic programming model with linear equality constraints and non-negative variables. Such a problem can be solved using first order optimization algorithm such as the gradient descent, or even more accurate algorithms such as the PDIP. This latter is largely discussed in the literature (for more details the reader can refer to Section V in [10]), where the authors have proposed a reduced PDIP algorithm in which modified Newton steps are used. It is characterized by a polynomial time compared to the NP-hard solver. The required arithmetic operations is of order $\mathcal{O}\left(M^{3}\right)$, where $M$ is the variable vector length defined in Section II, whereas the NP-hard requires a number of operations that increases exponentially with $M$ i.e. $\mathcal{O}\left(L^{M}\right)$.

Remark 2: It is worth noting that the equivalence between the $\ell_{0}$-norm and $\ell_{1}$-norm in $\left(P_{1}\right)$ hold only for the noiseless case as shown in [11], and not in our case. That is why performance loss are obtained as will be shown hereafter.

\section{B. Complexity order evaluation}

The computational complexity of the proposed problem is evaluated in Big-O notation, also called Landau's symbol, which is a well-understood symbolism widely used in complexity theory to describe the asymptotic behavior of functions [12], [13]. Basically, it tells how fast a function grows or declines. Table I summarizes the complexity order of the proposed detector, the simple MMSE, the MMSE-SIC proposed in [14], and the ML optimal detector. The ML based detector is NP-hard, thus it is the least computational cost efficient. The simple MMSE-based detector consists of a complex inversion of $N \times N$ matrix, and some matrix multiplications and additions. It is known to have a complexity of order $\mathcal{O}\left(N^{3}\right)$ as described in [15]. Regarding the MMSE-SIC, it has a computational complexity of $\mathcal{O}\left(N^{3}\right)+\mathcal{O}\left(M N^{2}\right)+\mathcal{O}\left(M^{2} N\right)$, 


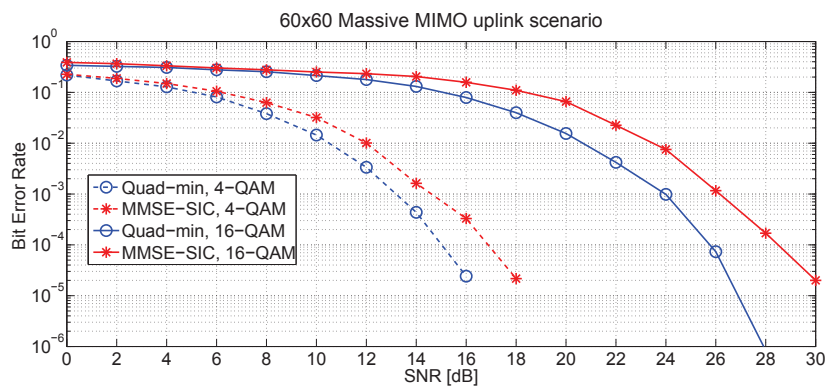

Fig. 2: BER performance comparison of the proposed detector versus the MMSE-SIC detector in large MIMO systems, when $N=M=60$.

which is equivalent to $\mathcal{O}\left(M^{3}\right)$ for determined systems, i.e. $M=N$. The [Quad-min] detector has the same order of complexity as the MMSE-SIC for determined MIMO systems. However, in overdetermined MIMO configuration and for $N \gg M$, the complexity order of the [Quad-min] becomes much lower than the MMSE-SIC. This is because the latter has a complexity that mainly depends on $N$ i.e. $\mathcal{O}\left(N^{3}\right)$, whereas the [Quad-min] complexity order, total and per iteration, depends only on $M[10]$.

\section{Simulation Results}

The performance of the proposed detector is compared to the MMSE-SIC in terms of Bit Error Rate (BER) in very large and massive MIMO systems. We consider a scenario where multiple UEs transmit a total streams $M$ via their antennas, and a BS equipped with $N_{r}$ receive antennas. The channel coefficients are i.i.d. circularly symmetric complex Gaussian distributed with zero mean and unit variance. The symbols are selected from 4-Quadrature Amplitude Modulation (QAM) and 16-QAM. For the implementation of the Quad-min detector, we use the cvx toolbox, which is a Matlabbased modeling system for convex optimization [16], [17]. We also use the Gurobi optimizer as a solver for our convex problem [18]. This solver is based on the PDIP methods for linear and quadratic programming. The solving algorithm can be implemented using the method proposed in [10].

\section{A. Comparison with other low-complexity detector}

Fig. 2 presents the configuration of a large MIMO determined system where the total streams and the number of receive antennas are equal to 60 . We compare the BER performance for both modulations 4-QAM and 16-QAM. When the total number of total is equal to the number of receive antennas i.e. very large dimensions with $M=N=60$, we observe that the Quad-min outperforms the MMSE-SIC over the whole SNR region, and better exploits the receive diversity through the joint detection. Assuming 4-QAM modulation constellation, the gain is about $1.7 \mathrm{~dB}$ at BER $10^{-2}$, and increases with the SNR growth to achieve almost $2 \mathrm{~dB}$ at BER $3.10^{-5}$. This gain becomes more important for 16-QAM constellation, it is about $3 \mathrm{~dB}$ at BER $10^{-2}$, and $3.7 \mathrm{~dB}$ at BER $10^{-2}$. The reason for the BER gain growth with the constellation size is that,

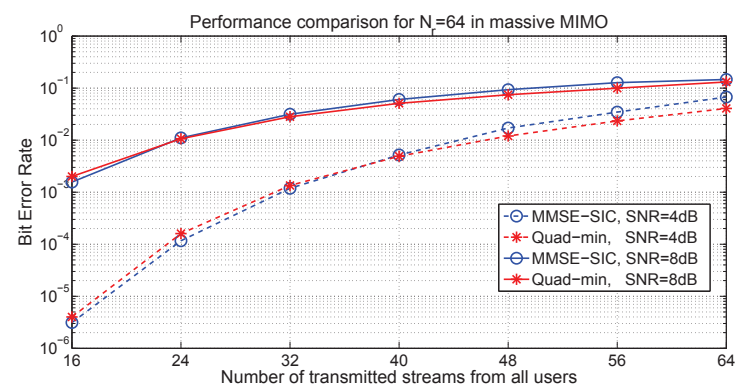

Fig. 3: BER performance comparison of the proposed detector versus the MMSE-SIC detector in massive MIMO.

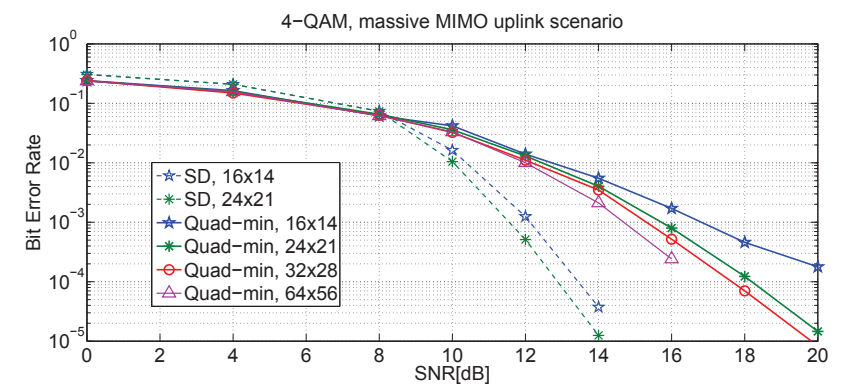

Fig. 4: BER performance comparison of the proposed detector with the sphere decoder in underdetermined systems.

the accuracy of the $\ell_{0}$-norm relaxation by the $\ell_{1}$-norm highly depends on the system dimensions and the sparsity threshold of the signal vector [11].

Next, we set $N_{r}=64$ and we consider 4-QAM modulation. In Fig. 3 we evaluate the BER performance with respect to the number of total streams. Both detectors yields similar performance for SNR $4 \mathrm{~dB}$. At $8 \mathrm{~dB}$ the proposed detector yields better performance when the number of total streams approaches $N_{r}$. Hence, for massive MIMO i.e. $N_{r} \gg M$, both low-complexity detectors performs similarly whereas for very large MIMO systems i.e. $N_{r}$ and $M$ of the same order, the Quad-min becomes more powerful. Furthermore, this latter represents stable performance for underdetermined systems whereas the MMSE-SIC must be modified because the required matrix inversion becomes unstable.

\section{B. Comparison with optimal detector in underdetermined sys- tems}

For underdetermined systems, Fig. 4 compares the BER performance of the proposed detection scheme to the sphere decoder (SD), described in [19]. We assume a 4-QAM constellation mapping known at both, the transmitter and the receiver. It can be observed that beyond $8 \mathrm{~dB}$, the $\mathrm{SD}$ outperforms the proposed scheme, e.g. at BER $10^{-2}$, a gain of about $2 \mathrm{~dB}$ and of $2.5 \mathrm{~dB}$ is obtained when the dimensions are $16 \times 14$ and $24 \times 21$, respectively. However, for the SD we cannot go beyond $24 \times 21$ dimensions due to a huge computational cost, whereas with the Quad-min it is possible to detect in a system with $N>90$ in less than one second for both constellation sizes as shown in Fig. 5. 


\begin{tabular}{|c|c|c|c|}
\cline { 2 - 4 } \multicolumn{1}{c|}{} & iteration number & computational cost per iteration & Total \\
\hline MMSE & 1 & $\mathcal{O}\left(N^{3}\right)$ & $\mathcal{O}\left(N^{3}\right)$ \\
\hline MMSE-SIC & 1 & $\mathcal{O}\left(N^{3}\right)+\mathcal{O}\left(M N^{2}\right)+\mathcal{O}\left(M^{2} N\right)$ & $\mathcal{O}\left(N^{3}\right)+\mathcal{O}\left(M N^{2}\right)+\mathcal{O}\left(M^{2} N\right)$ \\
\hline Quad-min & $\mathcal{O}(\sqrt{M})$ & $\mathcal{O}\left(M^{2.5}\right)$ & $\mathcal{O}\left(M^{3}\right)$ \\
\hline SD & 1 & $\mathcal{O}\left(\sqrt{L^{\gamma M}}\right)$ & $\mathcal{O}\left(\sqrt{L^{\gamma M}}\right)$ \\
\hline ML & 1 & $\mathcal{O}\left(\sqrt{L^{M}}\right)$ & $\mathcal{O}\left(\sqrt{L^{M}}\right)$ \\
\hline
\end{tabular}

TABLE I: Computational cost analysis

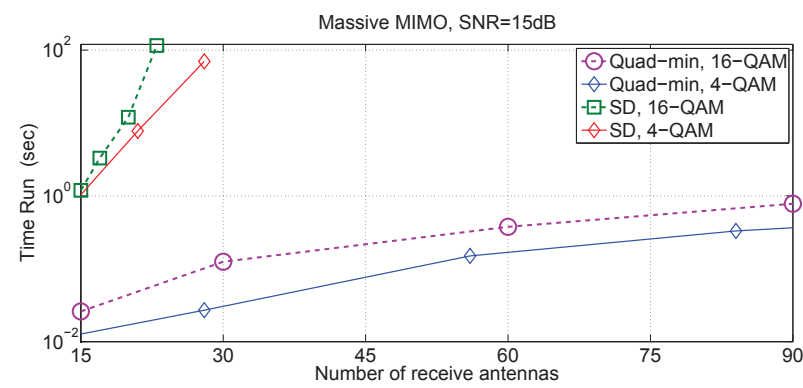

Fig. 5: Time-run comparison between the proposed detector and the sphere decoder.

Remark 3: It is important to mention that the relaxed constraints imposes the subvector components to be in the interval $[0,1]$, with their sum equal to one. Thus interpreting the solution subvector components as reliability values relative to the associated alphabet symbols, a preliminary analysis, which is still in progress, has shown that these output can be used to provide soft input to a channel decoder.

\section{CONCLUSION}

A new detection scheme has been proposed in the framework of massive and very large MIMO uplink scenario. It exploits the discrete constellation of the transmitted signals, and transforms the MIMO inputs model into a sparse model with inputs belonging to the binary set $\{0,1\}$. The optimal detection problem of the transformed model has been reformulated and relaxed based on well-known existing results in the sparse literature. In terms of performance and reliability, the proposed detector has been compared to the low-complexity MMSESIC detector, which offers a particularly interesting trade-off between complexity and performance. For high dimensions, it has shown a BER performance gain that increases with the signal dimension and depends on the input constellation, while keeping a same computational complexity order. However, a high performance dependency on both signal dimensions and sparsity threshold has been observed. The next steps are to seek new constraints that compensate the gap with the optimal detector, and to associate the proposed detector with a channel decoding scheme that should help in minimizing the error probability and should yield a more reliable transmission.

\section{REFERENCES}

[1] E. Telatar, "Capacity of multi-antenna gaussian channels," European Transactions on Telecommunications, vol. 10, pp. 585-595, 1999.
[2] F. Rusek, D. Person, B. Lau, E. Larson, T. Marzetta, O. Edfors, and F. Tuvesson, "Scaling up MIMO: Opportunities and Challenges with very Large Arrays," IEEE Signal Processing Magazine, vol. 30, no. 1, pp. 40-60, Jan. 2012

[3] X. Zhu and R. D. Murc, "Performance analysis of maximum likelihood detection in a mimo antenna system," IEEE Transactions on Communications, vol. 50, no. 2, pp. 187-191, Feb 2002.

[4] B. Hassibi and H. Vikalo, "On the sphere decoding algorithm i. expected complexity," IEEE Transactions on Signal Processing, vol. 53, no. 8, pp. 2806-2818, Aug 2005.

[5] J. Jalden and B. Ottersten, "On the Complexity of Sphere Decoding in Digital Communications," IEEE Transactions on Signal Processing, vol. 53, no. 4, pp. 1474-1484, April 2005.

[6] S. Boyd and L. Vandenberghe, Convex Optimization. Cambridge University Press, New York, 2004.

[7] L. Bai, C. Chen, and J. Choi, "Prevoting cancellation-based detection for underdetermined mimo systems," EURASIP J. Wirel. Commun. Netw., vol. 2010, pp. 96:1-96:11, Apr. 2010.

[8] D. Donoho, "For most large underdetermined systems of linear equations, the minimal L1 norm solution is also the sparsest solution," Comm. on Pure and Applied Mathematics, vol. 59, no. 6, pp. 797-829, June 2006.

[9] MIT Lecture, "Sparsity Based Regularization," http://www.mit.edu/ 9.520/spring10/scribe-notes/, 2010.

[10] D. Goldfarb and S. Liu, "An o (n 3 l) primal interior point algorithm for convex quadratic programming," Mathematical Programming, vol. 49, no. $1-3$, pp. $325-340,1990$.

[11] A. Aissa-El-Bey, D. Pastor, S. M. Aziz-Sbai, and Y. Fadlallah, "Recovery of Finite Alphabet Solutions of Underdetermined Linear System," IEEE Transactions on Information Theory, to appear.

[12] MIT Lecture, "Big O Notation," http://web.mit.edu/16.070/www/lecture/, 2003.

[13] E. Landau, Handbuch der Lehre von der Verteilung der Primzahlen. AMS Chelsea Publishing, 1909.

[14] B. Hassibi, "An efficient square-root algorithm for blast," in Proceedings IEEE International Conference on Acoustics, Speech, and Signal Processing, vol. 2. IEEE, 2000, pp. II737-II740.

[15] D. Wubben, R. Bohnke, V. Kuhn, and K.-D. Kammeyer, "Mmse extension of v-blast based on sorted qr decomposition," in Vehicular technology conference, 2003. VTC 2003-Fall. 2003 IEEE 58th, vol. 1. IEEE, 2003, pp. 508-512.

[16] M. Grant and S. Boyd, "CVX: Matlab software for disciplined convex programming, version 2.0 beta," http://cvxr.com/cvx, Sep. 2012.

[17] M. Grant and S. Boyd, "Graph implementations for nonsmooth convex programs," in Recent Advances in Learning and Control, ser. Lecture Notes in Control and Information Sciences. Springer-Verlag Limited, 2008, pp. 95-110.

[18] I. Gurobi Optimizatoion, "Gurobi optimizer reference manual," 2012. [Online]. Available: http://www.gurobi.com

[19] T. Cui and C. Tellambura, "An Efficient Generalized Sphere Decoder for Rank-Deficient MIMO Systems," IEEE Communications Letters, vol. 9, no. 5, May 2005. 